\title{
Review Paper: A Review of the Historical Evolutionary Process of Dry and Water Maze Tests in Rodents
}

\author{
Fahimeh Mohseni' ${ }^{1}$, Shahram Ghorbani Behnam² (D, Raheleh Rafaiee ${ }^{3^{*}}$ (D) \\ 1. Student Research Committee, School of Medicine, Shahroud University of Medical Sciences, Shahroud, Iran. \\ 2. Center for Health Related Social and Behavioral Sciences Research, Shahroud University of Medical Sciences, Shahroud, Iran. \\ 3. Department of Neuroscience, School of Advanced Technologies in Medicine, Mazandaran University of Medical Sciences, Sari, Iran.
}

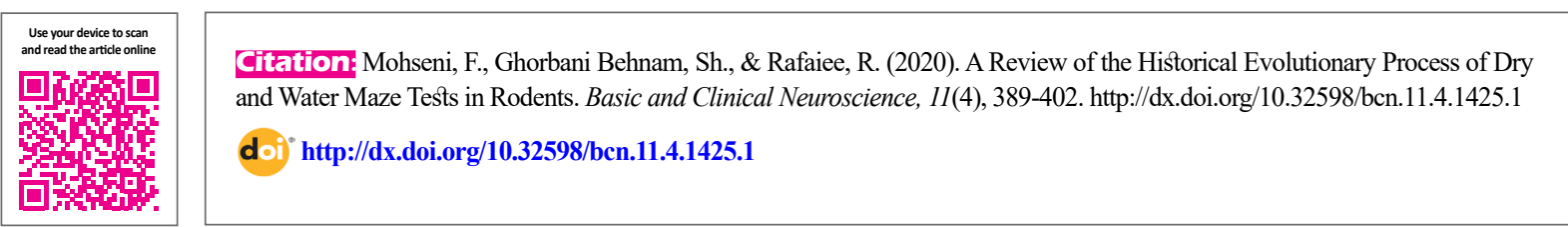

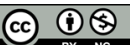

Article info:

Received: 05 Jul 2018

First Revision: 27 Jul 2018

Accepted: 16 May 2019

Available Online: 01 Jul 2020

Keywords:

Behavioral test, Maze,

Memory, Learning, History,

Rodents

\section{ABS T RACT}

This research provides an overview of the historical advances of the maze tests that are widely used to assess the cognitive impairments in rodents. Particularly, this study focuses on the issue of learning and memory behavioral tests, including dry and water mazes. Several types of mazes have been used in this setting, but their real advantages and applications depend on the type selected by the researcher. We answered some of the basic questions that any interested researcher in such studies may be faced with. The reviewed topics are as follows: the definition of maze learning, the role of the memory in the maze learning, the differences between several types of mazes, and foremost the rationale behind the maze constructions and designs.

\section{* Corresponding Author:}

Raheleh Rafaiee, MD, MPH, PhD.

Address: Department of Neuroscience, School of Advanced Technologies in Medicine, Mazandaran University of Medical Sciences, Sari, Iran. Tel: +98 (911) 1514422

E-mail: rahelerafaie@gmail.com 


\section{Highlights}

- Historical study is helpful to design a novel maze or to improve a previous one.

- Selection of the right maze can easily detect damage or improve memory.

- Choosing the different types of mazes depends on the aim of the research.

\section{Plain Language Summary}

The use of the mazes has a long history in learning processes of mammals. The well-trained animals solve the mazes rapidly without any considerable error. The novel mazes showed that the behavior of the animals in the mazes is flexible, thus, they can find the shortcuts if the previous path is blocked. To solve the maze, the subject must be able to construct a sequence of directions in its memory. According to a hypothesis, the shorter the distance from the reward, the more the tendency to solve the maze. Using a simple maze was easier and less time consuming. In most maze experiments, four common measures have been used to quantify the maze learning skill, including errors, time, distance, and the number of required trials. The maze design is probably the most challenging part of the maze experiment.

\section{Introduction}

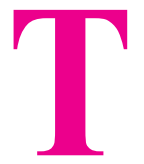

he use of the mazes has a long history in the studies of the memory and learning processes in mammals. In many physiological experiments, rodents, especially rats and mice are the model animals subjected to the maze experiments. Probably, the first maze experimentation was conducted by Willard S. Small at the beginning of the $20^{\text {th }}$ century. Small aimed to compare the mental abilities of the wild and tame white rats. To achieve this, he recorded the time and error factors for each rat when it was solving the Hampton Court maze (Small, 1901).

The maze learning has two aspects. When the animal is placed inside the maze for the first time, it begins to learn that the maze is a path to be traversed to get a reward (the discovery process). But solving the maze better than before (with fewer errors) is a distinct problem (the fixation process) (Simon, 1957; Yoshioka, 1929). The well-trained animals solve the mazes rapidly without any considerable error, so if the old path is blocked, they will be completely surprised. Following the pioneering experiments, the kinesthetic machine theory proposed that the behavior of the well-trained animals is inflexible and unthinking, in the maze. However, the subsequent experiments with novel mazes refuted this theory. They showed that the behavior of the animals in the mazes is flexible, thus, they can find the shortcuts if the previous path is blocked. Also, it has been proved that the rats have a strong spatial memory, and they can construct a plan of the maze in their minds (Olton, 1979).
To solve the maze, the subject must be able to construct a correct sequence of directions (i.e. left and right) in its memory and follow that sequence. The subject must choose the correct direction on the list, at any chosen point (Simon, 1957). Dennis and Henneman showed that the rat did not enter some of the cul-de-sacs in the first trial, and this avoidance of errors was not in a random manner (Dennis \& Henneman, 1932). However, the rats tend to explore their environment, thus, even if they choose one of two paths and get the reward in the first trial, it is more likely to choose the other path in the second trial (Olton, 1979). In a revolutionary study, Clark L. Hull from Yale University introduced the goal-gradient hypothesis to explain the subject's behavior in a typical maze. According to this hypothesis, the shorter the distance from the reward, the more the tendency to solve the maze (Hull, 1932). Simply, the term maze learning refers to a process in which the skill of the subject (here is a rat or mouse) in solving the maze is going better by more trials (Liggett, 1930).

Huang classified the affecting factors of the maze experiment into two groups: the conditions of the subject and the conditions of the maze (Huang, 1928). The applied type of maze depends on the research query (Olton, 1979). One of the most important pioneering maze experiments was performed by Karl S. Lashley who concluded that the data obtained using a simple maze was as valid as the data gathered with the complicated mazes commonly used in the previous studies. Also, using a simple maze was easier and less time consuming, so it provided the possibility of more trials (Lashley, 1918). Direction as a maze condition was classified into gen- 
eral and specific. For example, the maze rotation is about the general direction, and the relative position of the maze elements is about the specific direction. It has been shown that the rats learn the directions in the maze experiments (Yoshioka, 1929). Another maze condition is the reward. Furchtgott and Rubin concluded that the maze learning time is not a function of the magnitude of the reward when the incentive is above a defined threshold (Furchgott \& Rubin, 1953). This conclusion was in contrast with the conclusion of Cross, Rankin, and Wilson (Cross, Rankin, \& Wilson, 1964). Besides, the subject conditions include age (Oliverio \& Bovet, 1966; Spangler, Chachich, Curtis, \& Ingram, 1989), sex (Corey, 1930), and the degree of hunger (Washburn, 1926).

In most maze experiments, four common measures have been used to quantify the maze learning skill, including errors, time, distance, and the number of required trials to complete the maze learning process. Each of these measures has its advantages and disadvantages (Hunter \& Randolph, 1924). However, along with the development of various mazes, new measures have been used (Paul, Magda, \& Abel, 2009).

During the 1920s, studying the effects of different factors, including environmental and conditional factors, chemical agents, drugs, and foods on the memory and learning of the rodents using simple and complicated mazes gradually became a routine method in the field of behavioral physiology. In 1923, Carleton MacDowell published a study about the effects of alcoholism on the maze learning of white rats and their ascendants using the Watson circular maze (MacDowell, 1923; Watson, 1914). In the same year, Macht and Teagarden from Johns Hopkins University published a paper about the effects of the UV rays on the ability of rats to learn the circular maze (Macht \& Teagarden Jr, 1923). In 1927, a work was published by Calvin P. Stone and Marry Sturman-Huble. They compared the effects of food and sex incentives on the learning of two kinds of mazes: Asimple T-maze and a maze described by Warden (Stone \& Sturman-Huble, 1927; Warden, 1923).

Along with the rapid increase of such studies, some controversies were raised about the reliability and validity of the maze experiments, in the late 1920s. In 1927, Tolman and Nyswander investigated these two parameters for the maze measures of the rats. They concluded that the time, retracing, and perfect runs cannot provide reliable and valid scores for the individual abilities of rats to learn the mazes, but the number of errors can. The values of reliability coefficients are strongly dependent on the kind of applied maze (Stone \& Nyswander, 1927). In other words, the results of these experiments completely depended on the maze type.
Along with the initial development of the maze experiments, the mechanism of maze learning and the physiological basis of this process became a matter of query. Gradually, behavioral physiologists and neurobiologists focused on the functional neuroanatomy of maze learning. Such studies included a straightforward experimental design: making lesions in a specified part of the animal's brain and observing the results in maze learning. Using the albino rats, Lashley and Franz investigated the effects of the destructions of the different parts of the cerebral cortex on the maze learning ability (Lashley \& Franz, 1917). Since making lesions in the subcortical nuclei without damaging the cortical parts of the brain was very challenging at that time, the investigation of the effects of the subcortical lesions on the maze learning ability started two decades later (Brown \& Ghiselli, 1938).

According to what was said, the maze design/choice is probably the most challenging part of the maze experiment design. Thus, for studying the memory and learning phenomena using the mazes, the researchers must know the rationale behind the different types of mazes, the best type of maze for their experiment, and so on. Also, they should know how to design a completely novel maze or modify a previously described one if there is any necessity. It is important to note that the concepts of "maze pattern" and "maze construction" are different. The former term refers to the plan of alleys and blinds, while the latter refers to the physical structure of the apparatus (Chou, 1934). This review article aims to describe the various types of mazes that have been used in the studies of memory and learning in the rats and mice. Also, the history, rationale, and the principle of each type of mazes will be described in short. It is better to initially explain the principles of a typical memory and learning maze experiment, before talking about the different types of mazes, plans, and constructions.

\section{Memory System, Learning, and Navigation}

Memory is an information management system (Rolls, 2000). This system keeps the stimuli and brain states even after the diminishing and disappearing of the triggering event (Chaudhuri \& Fiete, 2016). Three main functions of this system include encoding, storage, and retrieval of the information. The memory system is a high-value evolutionary adaptation. Traditionally, memory is hypothetically divided into the short-term/working memory and the long-term/reference memory. The short-term memory provides the ability to remember the information for a few seconds to a few thousand seconds. The ability to remember the information for more than this period is defined as the long-term memory (Neath \& Surprenant, 2005). Also, 
the process during which the memory develops is called learning (Xu, Grigoryev, Li, Bian, Zhang, \& Liu, 2013).

One of the most valuable adaptive aspects of the memory system in mobile organisms is the ability to navigate their environment. The navigation is divided into allocentric or spatial navigation and egocentric navigation. The allocentric navigation depends on the distal cues; however, the egocentric navigation depends on the proximal and internal cues. Egocentric navigation is divided into route-based and path integration. In the route-based egocentric navigation, the subject follows a sequence of possible directions (for example, a sequence of left and right directions in a 2-dimensional plane), while in the path integration type of the egocentric navigation, the subject follows the vector integration to find the shortest path toward the goal. The direct sequence in the routebased navigation converts to automatic behavior and makes a part of the episodic memory (Vorhees \& Williams, 2016). The episodic memory consists of the ability to "the mental time travel" and review the past events (Hoerl, 2018).

The strategy based on the injuring a part of the brain and searching for the impairments in the maze learning abilities have shown that different neural networks are responsible for the allocentric and egocentric navigations. The hippocampus and entorhinal cortex are in charge of the spatial navigation, while the head direction cells of the separated parts of the brain are in charge of egocentric navigation. However, these two types of navi- gation are overlapped in some aspects, by partial neuroanatomical overlapping, such as the presence of the head direction cells in the hippocampus and entorhinal cortex (Vorhees \& Williams, 2016).

\section{Different Types of Mazes}

\subsection{Dry mazes}

\subsubsection{Modified Hampton Court maze}

The modified Hampton Court maze is the rectangular form of the Hampton Court garden maze, which is the ancestor of all types of the applied mazes up to now. The first application of this maze in the behavioral physiology refers to the initial work of Small in 1901 (Small, 1901). It seems that Small had not any scientific rationale to choose the Hampton Court maze, but he modified the maze to simplify the construction (Scott, 1931; Figure 1A).

\subsubsection{Watson circular maze}

This circular maze was presented by Johan B. Watson in 1914. This maze had a wooden base and concentric aluminum walls. The aluminum walls were mobile, so the openings and radial stops were possible to be repositioned. Then, it was possible to adjust the maze problem difficulty with the subject. Watson installed a camera lucida on his maze to easily record the animal movements (Watson, 1914; Figure 1B).
A

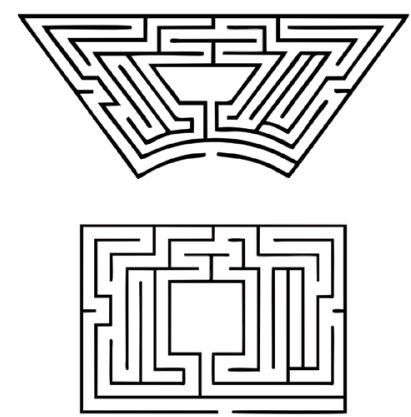

B

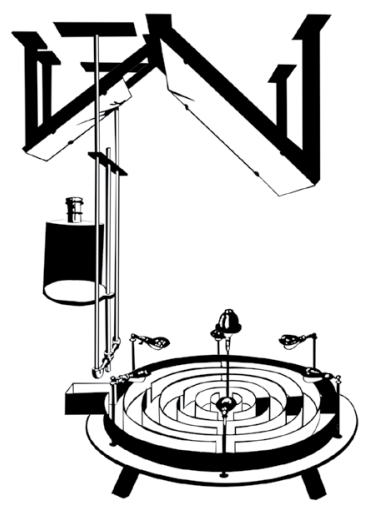

C

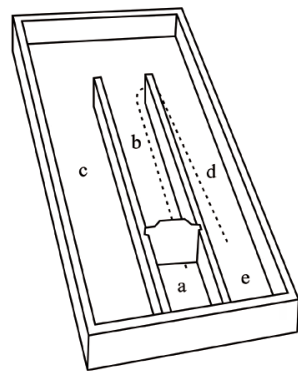

Figure 1. The modified Hampton Court maze

NEUR SCIENCE

A: The original pattern of the Hampton Court maze (right) and the rectangular form modified by small (left) (Scott, 1931). B: Watson circular maze equipped with the camera lucida (Watson, 1914). C: The simple maze; The subject putting on the starting section (a); When the gate opens and the animal passes the middle alley (b) it meets a choice. If it turns left, enters the cul-de-sac (c); and gets nothing. But if it turns right into the reward alley (d); it can find the reward place (e) (Lashley, 1918). 
A

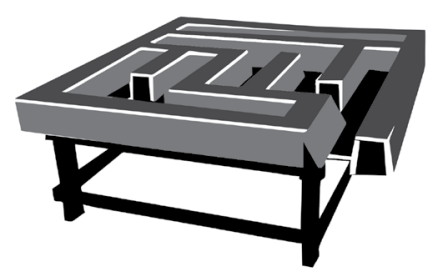

B

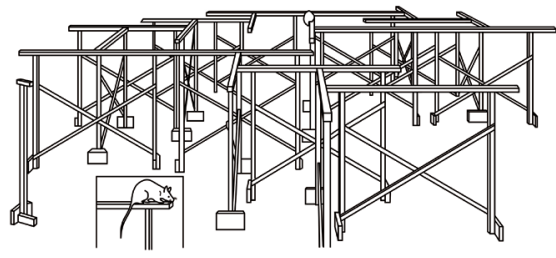

C

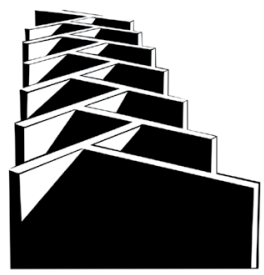

Figure 2. The elevated maze

A: First narrow-path elevated maze (Miles, 1927); B: Vincent elevated maze, the side walls are down (Miles, 1927); C: The block elevated maze (Dennis \& Henneman, 1932).

\subsubsection{The simple maze}

This maze was originally used in the studies of the behavior of the vertebrates other than rodents and found its way into the studies on rats by Lashley and Franz in 1917 (Lashley \& Franz, 1917). This is a very simple maze that comprises one cul-de-sac and one way leading to the reward (Figure 1C). It seems that the simplicity of this maze was the only reason for using it. As mentioned in the introduction, conducting a maze experiment with this maze is less time consuming than using a complicated maze, such as the Watson maze or the modified Hampton Court maze; however, the gathered data of both mazes had the same validity and reliability (Lashley, 1918).

\subsubsection{Elevated mazes}

\subsubsection{Vincent elevated maze}

The idea of the elevated maze was presented by Vincent in the middle of the 1910 s when he was trying to understand the role of the tactual sensation in maze learning. The paths of Vincent maze had no sidewall and were elevated two feet from the floor, but they were far enough to prevent rats from jumping between them (Figure $2 \mathrm{~A}$ ). According to this maze experiment, the tactualcutaneous sensation is the main sensation involved in the maze learning problem (Miles, 1927; Vincent, 1915).

\subsubsection{Narrow-path elevated maze (elevated skel- eton maze)}

This maze was devised in 1927 and had several differences from the Vincent maze. It comprised separate straight units that made any desirable pattern possible. The food or food box was not observable for the rat when it was running the paths. In contrast to the Vincent maze with 4-inch wide paths, the 1-inch wide paths were applied in this maze, yet, even the large rats could freely run and turn on the paths. The paths were supported by a few thin uprights (Figure 2B). The distances between
A

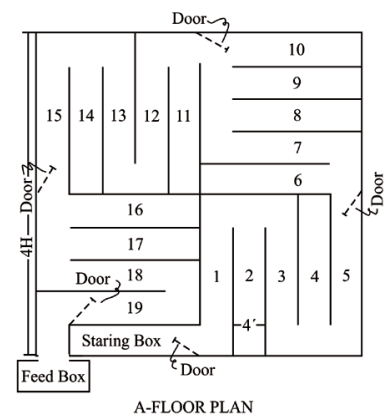

B

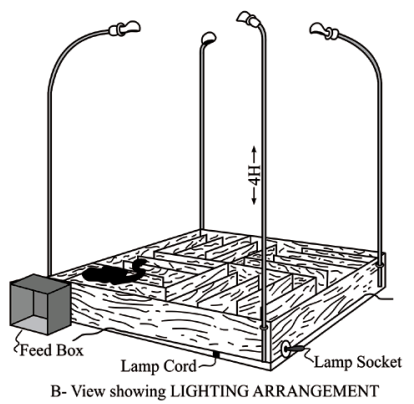

RANGEMEN

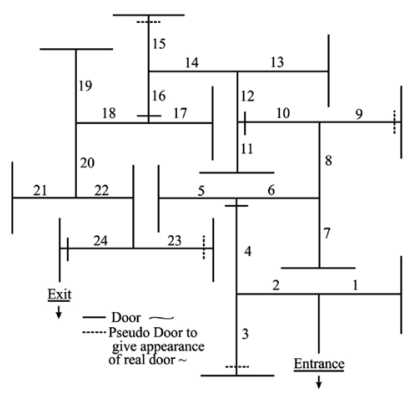

NEUR SCIENCE

Figure 3. The Modified Carr maze

A and B: Modified Carr maze used by Calvin P. Stone (Stone, 1928); C: The plan of the multiple T-maze presented by Stone and Nyswander (Stone \& Nyswander, 1927). 
A

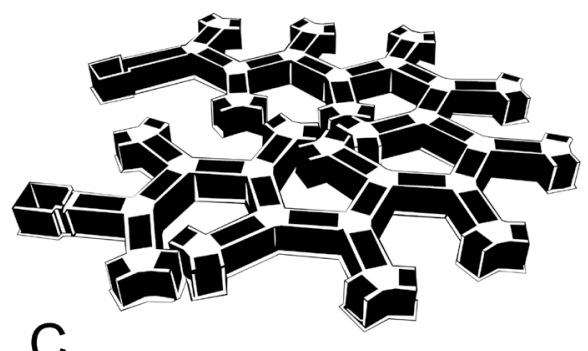

C

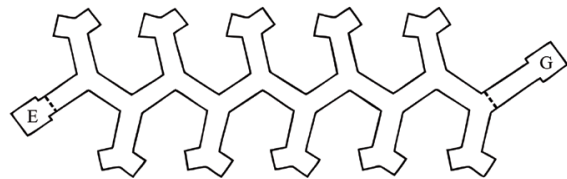

B

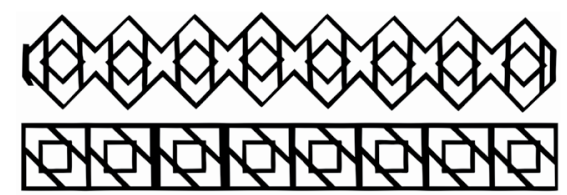

D

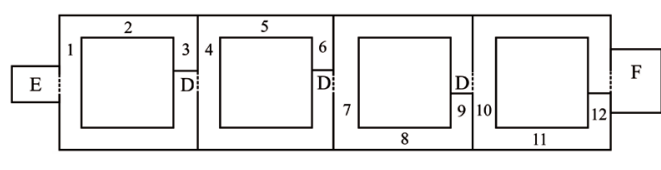

Figure 4. Warden's Y-maze

NEUR SCIENCE

A: Warden-Warner maze. A multiple Y-maze consisting of the Y-shaped units, entrance box, and the end box. Different maze patterns can be made by assembling the Y-shaped units (Warden, 1929); B: The linear pattern of Warden-Warner Maze (Warden, 1929); C: Two different linear mazes. Corner to corner arranged maze (X-maze) (top) is more difficult than the side to side arranged maze (below) (Miles, 1931). D: Liggett unit maze; E: The entrance box; D: The gates between two sections; F: The food box. The numbers represent the alleys. Each section has only a blind and a right way (Liggett, 1930).

the parallel paths were 18 inches. Miles named this maze as "the elevated skeleton maze". All these modifications let animals to efficiently apply vision along with other sensations (Miles, 1927).

\subsubsection{The block elevated maze}

This maze was devised by Dennis as a modification of the elevated skeleton maze, in 1931. As shown in Figure $2 \mathrm{C}$, He replaced the structure of the uprights-base blocks by the solid blocks. Since each block was a separate unit, it was possible to construct any desired pattern by these blocks. The block elevated maze had some advantages over the elevated skeleton maze: 1 . It wasn't custombuilt; 2 . It was more stable than the elevated skeleton maze; 3. The handling of the block units was easier; 4 .
It was possible to use any edges of the block units as paths; 5 . It was possible to polish the edges to clean up any traces of the previous runs; and 6. The units of this maze were more similar than that of the elevated skeleton maze. The only advantage of the elevated skeleton maze over the block elevated maze was the fewer wide paths, but it could be overcome by attaching the block to the floor (Dennis \& Henneman, 1932).

\subsubsection{Elevated plus maze}

In 1985, Montgomery did an interesting observation. He observed the rats exposed to an elevated (open) maze were more prone to show retreats, compared with the rats exposed to an enclosed maze. Then, Montgomery used a maze with both open and enclosed alleys to investigate this
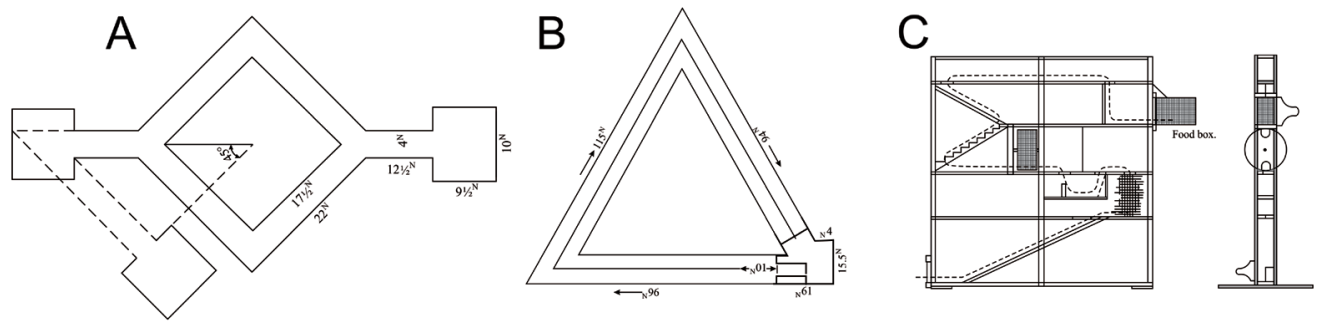

Figure 5. Yoshioka's mazes

A and B: The Yoshioka's mazes. The diamond maze (a) and triangular maze (b) (Yoshioka, 1929). C: Two different views of the Walton vertical maze. The dotted line represents the true path toward the food that is in a wire cage (Walton, 1930). 


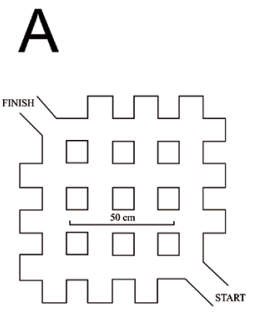

B

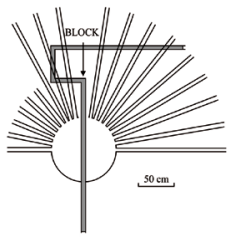

C

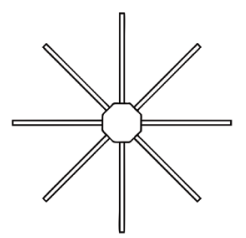

D

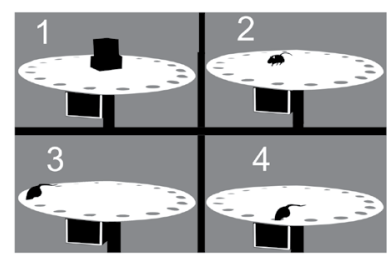

NEUR SCIENCE

Figure 6. Dashiell's open arena maze

A: Dashiell maze (Olton, 1979); B: Sunburst maze. The shaded path is the path in which rats were trained (Olton, 1979); C: The plan of the radial arm maze (Olton \& Samuelson, 1976); D: The different stages of the Barnes maze experimentation: 1: Before starting the trial the rat is in the start box; 2: The trial starts with removing the start box; 3 : An error by the rat; and 4: The successful ending of the trial by the rat (Barnes, 1979).

behavior. Rats preferred the enclosed alleys than the open alleys. This research showed that this type of maze experiment can be a possible method to investigate the anxiety in rats (Pellow, Chopin, File, \& Briley, 1985). Itoh and colleagues showed that the latency of entering the enclosed arm from the open arm decreased by 10 seconds or more when trials were repeated the next day. Therefore, these variables have been introduced for the assessment of learning and memory (Itoh, Nabeshima, \& Kameyama, 1990).

\subsubsection{Carr maze}

This maze was presented by Harvey A. Carr in 1917. The maximum application of this maze in rat studies was reported until 1928 when Calvin P. Stone explained why even a modified version (Figure $3 \mathrm{~A}$ and $\mathrm{B}$ ) of this maze is not appropriate for research (Stone, 1928).

\subsubsection{T-maze}

The first application of a T-maze in the studies of rats refers to a work by Hunter in 1920 . He presented a multi- ple T-maze with 10 choice points. The continuous correct path in this maze was an alternation series of right and left turns. This maze was called "spatial maze" (Shepard, 1920). In 1924, Hunter and Randolph concluded that a simple T-maze greatly reflects the individual differences in the learning ability. By that time, the simple T-maze was the most reliable maze for the study of the habit formation in white rats (Hunter \& Randolph, 1924).

Stone and Nyswander reported multiple T-maze experiments upon rats, in 1927. One of the most interesting characteristics of their maze was the unidirectional doors that made retracing partly impossible, so the exploration behavior was possible by then (Figure 3C). They have applied this maze to compare four different methods of calculating the reliability of learning scores (Stone \& Nyswander, 1927).

\subsubsection{Temporal maze}

In 1920, Hunter devised a maze consisted of a rectangular continued path and a cross path between the two parallel
A

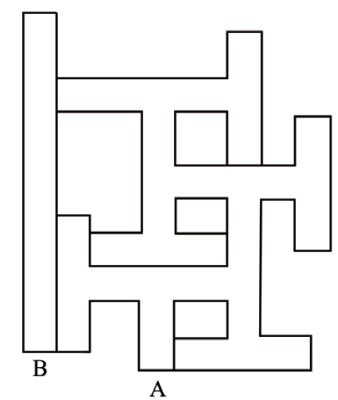

B

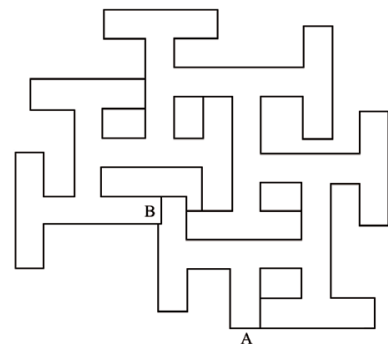

C

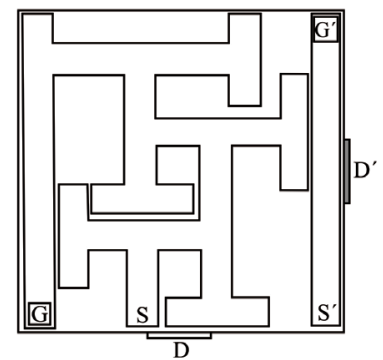

NEUR:SCIENCE

Figure 7. Multiple-T water maze

A: Biel maze; B: Cincinnati maze. The points A and B represent starting and landing points, respectively (Vorhees, 1987). C: The pattern of the Biel maze. The points $S$ and $G$ represent the starting point and the goal landing, respectively. The $S^{\prime}$ and $G^{\prime}$ are the training counterpart paths for $\mathrm{S}$ and $\mathrm{G}$. The $\mathrm{D}$ and $\mathrm{D}$ ' represent the doors of the main pathway and training pathway, respectively (Biel, 1940). 


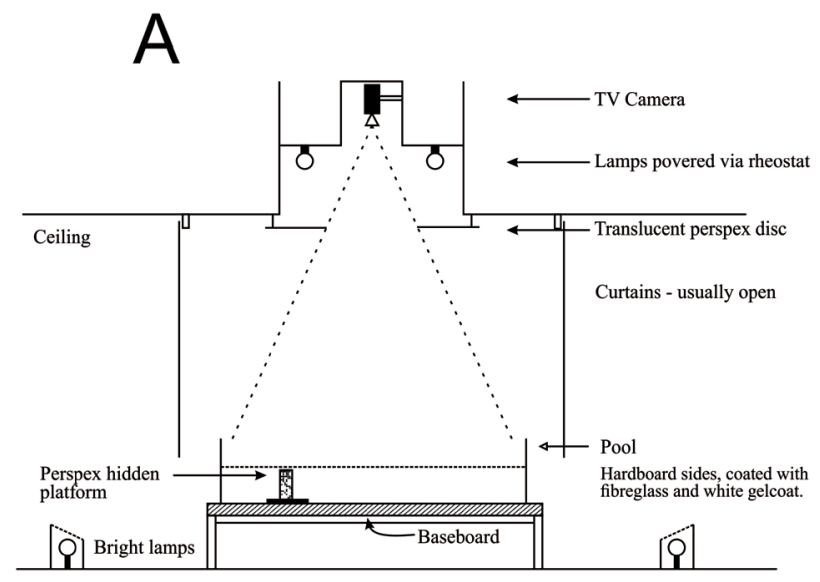

B

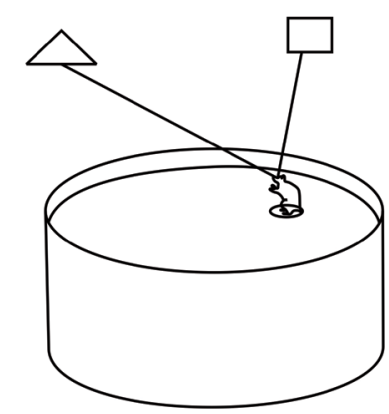

Figure 8. Maze with no proximal cues for spatial memory

NEUR SCIENCE

A: The lateral cross-section of the Morris water maze (Morris, 1984); B: The schematic picture.

sides of the rectangle. The starting point was embedded at one end of the cross path. It was possible to close one side of the rectangle path. Before the animal running, there was a block just behind the start point, so moving back was impossible for the animal. When the animal entered the open returning path, the block was shifted toward the other returning path, thus, the animal was allowed to complete the circuit and return to the starting point. Hunter used this maze to investigate the learning of the alternation series in white rats (Shepard, 1920).

\subsubsection{Warner-Warden maze}

In 1929, Warden presented a maze consisting Y-shaped units (multiple Y-maze; Figure 4A); later, it was named as Warden-Warner maze. The angles of the choice points differ between the multiple Y-maze and the multiple T-maze. In the T-maze, the angle of any choice point is 90 degrees, so it is impossible to make two successive turns toward the one direction. However, any successive turn toward the same direction is possible in the Y-maze (Warden, 1929).

\subsubsection{Unidirectional mazes}

Warden presented a linear maze derived from the multiple Y-maze to investigate serial learning (Figure 4B). This maze had a symmetrical and tortuous pattern. Warden proposed this pattern for studying the effects of the maze length on the learning variables (Warden, 1929). It has been found that for the rats the solving of a unidirectional maze is more difficult than the solving of a bidirectional maze (Miles, 1931). Figure 4C represents two different types of unidirectional mazes. In 1930, Liggett devised a linear maze to overcome the problem of the variable difficulty of the different blinds of the maze ap- paratus; he named it "the unit maze". The retracing was made impossible in this maze. This change increased the reliability coefficients of this maze, compared with the ordinary mazes (Liggett, 1930). Figure 4D presents the Liggett's maze.

\subsubsection{The tunnel maze}

In 1929, Trueblood invented a maze to study the behavior of the rats in a rotating maze. It was called "tunnel maze," because rats run through a tunnel with the wooden sides, a ground glass floor, and a ground glass ceiling. Also, the electric lights were installed under the paths made of the ground glass, thus, the animal movements were observable through the shadows on the ceiling made of the ground glass. However, the animal could not get any visual cues (Trueblood, 1929).

\subsubsection{Diamond and rectangular mazes}

In 1930, Yoshioka invented two different mazes to verify the direction orientation of the albino rats. One maze was called "diamond maze," which was a straight path divided into two same length alleys by an angle of 90 degrees. Then, the alleys turned toward the straight path by an angle of 90 degrees and met each other to complete a square. The position of the food box was variable relative to the central axis of the maze. Also, the food box was possible to be rotated in the same plane in which the maze path was rotating (Figure $5 \mathrm{~A})$. This maze was better than a T-maze to verify direction orientation. At the choice point of a typical T-maze, the rat met a plane wall that did not provide many sensory cues to indicate that there existed the right and left paths. Contrary, in the diamond maze, the rat can readily distinguish the right and left paths, even before arriving at the choice point 
Another maze devised by Yoshioka was an "equilateral triangular maze" in which both paths begin as common paths that are separated after 10 inches by a partition wall (Figure 5B). The flip-flop doors were installed at the openings of the separate paths; thus, it was possible for one path to be opened and the other to be closed at the same time. Yoshioka applied this maze to investigate the relationship between the direction of the orientation at a typical choice point and the direction of turning orientation at the end of a typical blind. He made the habit by trials in which one path was blocked and the other opened alternatively. Then, he carried out the trials in which both paths were opened. Also, he broke the habit by blocking the preferred path and observed a positive relationship between the direction of orientation at a typical choice point and the direction of turning orientation at the end of a typical blind (Yoshioka, 1929).

\subsubsection{Vertical maze}

In 1930, Albert Walton presented a vertical (or reversal) maze for the lecture-room demonstration (Walton, 1930) (Figure 5C).

\subsubsection{Dashiell maze}

In 1930, Dashiell introduced an open arena maze in which several similar blocks were placed in the middle by equivalent distances between them; thus, so many alleys with the same distance to the goal were created (Figure 6A). Dashiell aimed to investigate the exploration behavior in rats. Dashiell observed that the rats that learned the maze well preferred to try new paths. Other mazes that were devised to study the exploration behavior of rats were Bättig hexagonal and Barnett residential mazes (Dashiell, 1930; Olton, 1979).

\subsubsection{Sunburst maze}

When the tendency toward the exploration behavior was confirmed in rats, the hypothesis of the cognitive map would be raised. According to this hypothesis, rats can find shortcuts toward the goal (if there was any shortcut) by constructing a spatial map in their minds. One of the best experiments investigated this theory was conducted by Tolman and his colleagues (Tolman, Ritchie, \& Kalish, 1946). They designed a maze experiment wherein the rats were trained to run into a simple but indirect path with no blind toward the food. Then, the simple path was blocked and the rats were reinforced to choose a path among a lot of arm alleys (Figure 6B). This maze is known as the sunburst maze (Olton, 1979).

Tolman et al. observed that most rats chose the alley toward the goal, in the first trial. They concluded that the rats can understand the spatial position of the start point concerning the goal. However, these results were not repeatable; with the hope to bypass the block, choosing an alley adjacent to the training path could be a logically right choice (Olton, 1979).

\subsubsection{Radial arm maze}

To assess the place learning in rats, Olton and Samuelson designed an experiment in 1976, including an apparatus known as radial arm maze. This maze offered only one choice point (in one special spatial location) to rats to choose among eight distinctive but equivalent alleys (Olton \& Samuelson, 1976) (Figure 6C).

This maze was one of the best mazes developed for studying spatial memory and working memory. Olton and Samuelson aimed to design a maze to investigate the spatial memory without any interference from proximal cues. However, this purpose was not completely achieved, because of the presence of odors, and the ability of rats to use other proximal intra-maze cues right after entering any chose alley (Hyde, Hoplight, \& Denenberg, 1998; Olton, 1979; Olton \& Samuelson, 1976). The problem was overcome (35) by the development of a revolutionary maze method: The Barnes maze, 1979.

\subsubsection{Barnes maze}

Two years after the work of Olton and Samuelson, Carol A. Barnes presented maze experimentation and an apparatus to solve the problem of the intra-maze cues in the studies about spatial memory. Barnes was asking for the differences in cognitive abilities between young and old rats. The Barnes maze was composed of a circular platform (1.22 $\mathrm{m}$ in diameter) with 18 circular holes $(9.5$ $\mathrm{cm}$ in diameter) evenly distributed around the edge of the platform. Also, a tunnel was placed below the platform and was merely accessible from the hole above it. Both the circular platform and the tunnel were rotatable.

Before every trial, the rat was held into the tunnel for about four minutes, then, returned to the home cage for one minute. At the start of every trial, the rat was held in the center of the platform into an open-ended cylinder for 30 seconds, after, the cylinder was dragged $6 \mathrm{~m}$ upward and the rat was left in the center of the platform. The rat started searching for the hole upon the entrance of the tunnel to escape from an intense light provided by two projectors. A video camera $6 \mathrm{~m}$ above the circular platform recorded the movements and latency of the rat. Bending into any wrong hole was considered as an error (Figure 6 D). Besides, the rat was unable to use the proxi- 
mal cues to escape, because of the rotation of the platform and the tunnel between the trials, and the separation of the platform and the rest of the room space using partition screens. So, the rats should be only relied on their spatial memory to find the entrance of the tunnel (Barnes, 1979).

Today, the Barnes maze is known as the best dry maze for assessing the spatial memory of rats and mice. After the introduction of the initial version of this maze by Barnes, some modified versions of this maze were developed (Rosenfeld \& Ferguson, 2014).

\subsection{Water mazes}

The first application of a water maze refers to an experiment by William McDougall. He tried to test the wellknown hypothesis of Lamarck and designed an experiment that included a maze apparatus. Initially, he used a dry maze, but it was very time-consuming to teach the rat to solve the maze and get a reward. Thus, McDougall replaced the dry maze by a water maze in which the rats were to escape naturally. He replaced the food incentive with the incentive to escape from the water. Also, this innovative idea was to overcome the problem of uncertainty about the degree of hunger (McDougall, 1927). The results of the maze learning experiments derived by the food incentives depend on variables (such as sex, age, etc.) other than that of the experiments derived by the water escape and aversion escape incentives (Hodges 1996; Wenrick, 1935) using environmental visuospatial cues. However, maze tasks differ along many dimensions, including 1 . Indeed, the incentive of the water maze is aversion-based, conversely, the dry maze includes the reward-based incentive. Also, it is impossible to use the starvation incentive for learning the maze in some studies (Wenrick, 1935). However, some cautions must be concerned about using the water mazes; some factors, such as the water temperature can affect the results (Caldwell \& Mosman, 1951).

After the above-mentioned work of McDougall, the water mazes gradually found their way into the maze experiments, initially as constructions based on the previously designed patterns for the dry mazes. Later, some maze patterns were designed specifically for applying to the water maze construction.

\subsubsection{Biel maze}

In 1940, William C. Biel used a multiple-T water maze to unravel the developmental adaptability of young rats (Figure 7A and C) (Biel, 1940). This maze included a straight path for training and measuring the swimming abilities in rats. Individual differences, such as sex and body weight did not affect the learning of this maze. This was a valuable maze in assessing the effects of different agents and brain lesions on the cognitive ability of rats, especially in prenatal developmental stages. The coefficient of detection of the Biel maze has been reported $10 \%$ to $20 \%$ (Vorhees, 1987 ).

\subsubsection{Cincinnati water maze}

In 1987, Charles V. Vorhees from the University of Cincinnati introduced an improved version of the Biel maze to increase the sensitivity of the water maze experiments. These two mazes differed in terms of complexity, scale, and construction materials. The Cincinnati water maze was twice complex, compared with the Biel maze. Also, the detection power of the Cincinnati maze in the lower doses of a standard toxic agent was more than that of the Biel maze (Vorhees, 1987; Figure 7B).

\subsubsection{Morris water maze}

In 1981, Richard G. M. Morris from the University of St Andrews devised a maze that did not offer any local cue to the goal. The apparatus included a circular water pool (1.30 $\mathrm{m}$ in diameter and $0.6 \mathrm{~m}$ in height), a white mobile platform $(0.11 \mathrm{~m}$ in diameter), and a closed-circuit video camera on top. The pool was filled with water up to $0.4 \mathrm{~m}$. The tip of the platform was $1 \mathrm{~cm}$ below the level of water. Water was made opaque by adding some milk so that the white platform was hidden. Therefore, the rats received no proximal cue from the platform, and in the first trial, they were forced to search the pool (randomly) to find a basement for landing. In the next trials, they have only relied on their spatial memory and no proximal cue to find the landing platform (Morris, 1984; Morris, 1981). This was the first useful maze to investigate the spatial memory without any interfering by the proximal cues and choice points. The invention of this maze started a revolution in the study of spatial memory (Figure 8).

The above-reviewed literature is a short description of the initial development of the maze experiments in the studies about the different aspects of memory in rodents. The rationale behind the shifting interests from one type of maze to another can be deduced, according to the previous sections. As mentioned in the Introduction, there is no competition between different types of mazes, and choosing a maze is mostly dependent on the research aim. For example, both Morris water maze and Barnes maze tasks assess learning, working memory, and spatial memory. However, when the researchers have to choose between them they should be 
aware that the former induces more stress and needs more physical effort to perform than the later (Vargas-López, Lamprea, \& Múnera, 2011).

Some mazes are better for studying a special concern than the others. For example, consider two multiple T-mazes with the same constructions and patterns, but differ in the number of T-units. In this case, the favorite is the one that gives better validity and reliability coefficients. The results of comparative studies concerned about validity and reliability coefficients have provided a basis to regard bias between some of the mazes. Also, the low validity and reliability of some primary mazes incite the development of the new mazes. The study of maze learning using a special type of cue (when the presence of other types of cues is interfering) rises another challenge for the researchers. They have to choose the better maze among two or more mazes designed for the same purpose. The development of the Barnes maze and Morris water maze for the assessment of the spatial memory are the classic examples of such situations.

The development of the maze tasks is similar to the development of any pieces of technology in the modern world. The development of these tasks has been started with the primary versions and included some problems in assessing the different aspects of learning and memory, then, the modified versions were developed. Today, many modified versions of some of the initial mazes are available (for example, see Vargas-López et al., 2011; Dobson et al., 2012).

Behavioral physiology and neuroscience owe a lot to the maze tasks, for what these tasks provided to assess learning and memory in the last century. Currently, the neuroanatomical maps of the brain of the rats and mice are available, in which the parts in charge of many aspects of learning and memory are defined. For example, the hippocampus and less importantly striatum are the organs in charge of the spatial memory, so injuries in these parts of the brain can affect spatial navigation (D’Hooge \& De Deyn, 2001). Besides, different parts of the neocortex, including the frontal cortex, prefrontal cortex, anteromedial extrastriate visual cortex, primary visual cortex, posterior parietal cortex, and somatosensory cortex are involved in the learning of the water mazes (Hoh, Kolb, Eppel, Vanderwolf, \& Cain, 2003).

The maze tasks can be paired with functional brain imaging and histochemical methods to provide the more precise functional neuroanatomy of the brain. As an example, such studies have revealed the role of the cerebellum in the working memory and less important the spatial memory (Lalonde \& Strazielle, 2003).
According to the present review, we can deduct that the history of the use of mazes in the studies of learning and memory backs to more than a century ago. Thousands of works have been published in this issue, but maze tasks are actually at the beginning of a long way. The maze tasks in conjugation with other methods are going to answer one of the mysterious questions ever: "how does the brain work as a complex system?"

The current study faced some limitations for comparing the initial and recent mazes. Initial mazes were designed when there was not much scientific knowledge on the memory system, the phases of memory, learning process, and related brain structures. These mazes were designed to study the whole (not a part of, nor the classified process) brain processes. Therefore, comparing the old mazes with new types in terms of the assessment of memory types and memory phases was not feasible in this study.

Future studies can compare the new mazes in terms of application (which are more commonly used; which interventions they are usually used for), and discuss the memory types (working/reference), related brain structures, and phases that can be studied using the mazes.

\section{Ethical considerations}

\section{Compliance with ethical guidelines}

This article did not contain any studies performed with animals and all ethical principles are considered in this article.

\section{Funding}

This research did not receive any grant from funding agencies in the public, commercial, or non-profit sectors.

\section{Authors' contributions}

Investigation, writing - review \& editing: Fahimeh Mohseni; Formal analysis, resources, data curation, writing - original draft preparation: Shahram Ghorbani Behnam; Writing - review \& editing, vsualization, supervision: Raheleh Rafaiee.

\section{Conflict of Interest}

The authors declared no conflict of interest.

\section{Acknowledgments}

We thank Dr Mehdi Khaksari, the manager and head of the Addiction Studies Department for his comments on an earlier version of the manuscript. 


\section{References}

Barnes, C. A. (1979). Memory deficits associated with senescence: A neurophysiological and behavioral study in the rat Journal of comparative and Physiological Psychology, 93(1), 74-104. [DOI:10.1037/h0077579] [PMID]

Biel, W. C. (1940). Early age differences in maze performance in the albino rat. The Pedagogical Seminary and Journal of Genetic Psychology, 56(2), 439-53. [DOI:10.1080/08856559.1940.10534511]

Brown, C. W., \& Ghiselli, E. E. (1938). Subcortical mechanisms in learning. II. The maze. Journal of Comparative Psychology, 26(1) 27-44. [DOI:10.1037/h0055581]

Caldwell, W. E., \& Mosman, K. F. (1951). The role of temperature change as reinforcement. The Journal of Psychology, 32(2), 231-9. [DOI:10.1080/00223980.1951.9916092]

Chaudhuri, R., \& Fiete, I. (2016). Computational principles of memory. Nature Neuroscience, 19(3), 394-403. [DOI:10.1038/ nn.4237] [PMID]

Chou, S. K. (1934). A water elevated maze. The Journal of General Psychology, 11(1), 223-6. [DOI:10.1080/00221309.1934.9917833]

Corey, S. M. (1930). Sex differences in maze learning by white rats. Journal of Comparative Psychology, 10(4), 333. [DOI:10.1037/h0071778]

Cross, H. A., Rankin, R. J., \& Wilson, J. (1964). Influence of amount of reward on maze learning in hooded and albino rats. Psychonomic Science, 1(1-12), 275-6. [DOI:10.3758/ BF03342908]

D'hooge, R., \& De Deyn, P. P. (2001). Applications of the Morris water maze in the study of learning and memory. Brain Research Reviews, 36(1), 60-90. [DOI:10.1016/S01650173(01)00067-4]

Dashiell, J. F. (1930). Direction orientation in maze running by the white rat. Comparative Psychology Monographs, 1930, 7(2) 71-2. https:// psycnet.apa.org/record/1930-02625-001

Dennis, W., \& Henneman, R. (1932). The non-random character of initial maze behavior. The Pedagogical Seminary and Journal of Genetic Psychology, 40(2), 396-405. [DOI:10.1080/08856559.1 932.10532433]

Dobson, C. C., Mongillo, D. L., Poklewska-Koziell, M., Winterborn, A., Brien, J. F., \& Reynolds, J. N. (2012). Sensitivity of modified Biel-maze task, compared with Y-maze task, to measure spatial learning and memory deficits of ethanol teratogenicity in the guinea pig. Behavioural Brain Research, 233(1), 162-8. [DOI:10.1016/j.bbr.2012.04.042] [PMID]

Furchgott, E., \& Rubin, R. D. (1953). The effect of magnitude of reward on maze learning in the white rat. Journal of Comparative and Physiological Psychology, 46(1), 9-12. [DOI:10.1037/ h0055050] [PMID]

Hodges, H. (1996). Maze procedures: The radial-arm and water maze compared. Cognitive Brain Research, 3(3-4), 167-81. [DOI:10.1016/0926-6410(96)00004-3]

Hoerl, C. (2018). Episodic memory and theory of mind: A connection reconsidered. Mind and Language, 33(2), 148-60. [DOI:10.1111/mila.12170]

Hoh, T. E., Kolb, B., Eppel, A., Vanderwolf, C., \& Cain, D. P. (2003). Role of the neocortex in the water maze task in the rat: a detailed behavioral and Golgi-Cox analysis. Behavioural Brain Research, 138(1), 81-94. [DOI:10.1016/S0166-4328(02)00237-1]

Huang, L. (1928). An analysis of the maze technique. Journal of Comparative Psychology, 8(4), 301-11. [DOI:10.1037/h0069773]

Hull, C. L. (1932). The goal-gradient hypothesis and maze learning. Psychological Review, 39(1), 25. [DOI:10.1037/h0072640]

Hunter, W. S., \& Randolph, V. (1924). Further studies on the reliability of the maze with rats and humans. Journal of Comparative Psychology, 4(4), 431-22. [DOI:10.1037/h0073265]

Hyde, L. A., Hoplight, B. J., \& Denenberg, V. H. (1998). Water version of the radial-arm maze: Learning in three inbred strains of mice. Brain Research, 785(2), 236-44. [DOI:10.1016/ S0006-8993(97)01417-0]

Itoh, J., Nabeshima, T., \& Kameyama, T. J. P. (1990). Utility of an elevated plus-maze for the evaluation of memory in mice: effects of nootropics, scopolamine and electroconvulsive shock Psychopharmacology, 101(1), 27-33. [DOI:10.1007/BF02253713] [PMID]

Lalonde, R., \& Strazielle, C. (2003). The effects of cerebellar damage on maze learning in animals. The Cerebellum, 2(4), 300 [DOI:10.1080/14734220310017456] [PMID]

Lashley, K. (1918). A simple maze: With data on the relation of the distribution of practice to the rate of learning. Psychobiology, 1(5) 353-67. [DOI:10.1037/h0075008]

Lashley, K. S., \& Franz, S. I. (1917). The effects of cerebral destruction upon habit-formation and retention in the albino rat. Psychobiology, 1(2), 71-139. [DOI:10.1037/h0071400]

Liggett, J. R. (1930). A study of maze measures and of the factors involved in maze learning. The Pedagogical Seminary and Journal of Genetic Psychology, 38(1-4), 78-90. [DOI:10.1080/08856559.1930.10 532252]

MacDowell, E. C. (1923). Alcoholism and the behavior of white rats II. The maze-behavior of treated rats and their offspring. Journal of Experimental Zoology Part A: Ecological Genetics and Physiology, 37(5), 417-56. [DOI:10.1002/jez.1400370503]

Macht, D. I., \& Teagarden Jr, E. J. (1923). The effect of ultra-violet rays on rats in the circular maze. Proceedings of the Society for Experimental Biology and Medicine, 20(7), 387. [DOI:10.3181/00379727-20-187]

McDougall, W. (1927). An experiment for the testing of the hypothesis of Lamarck. British Journal of Psychology, 17(4), 267-304 [DOI:10.1111/j.2044-8295.1927.tb00432.x]

Miles, W. R. (1927). The Narrow-Path Elevated Maze for Studying Rats. Proceedings of the Society for Experimental Biology and Medicine, 24(5), 454-6. [DOI:10.3181/00379727-24-3414

Miles, W. R. (1931). Unidirectional mazes for use with animals. The Journal of General Psychology, 5(2), 275-8. [DOI:10.1080/00221309.1 931.9918400]

Morris, R. (1984). Developments of a water-maze procedure for studying spatial learning in the rat. Journal of Neuroscience Methods, 11(1), 47-60. [DOI:10.1016/0165-0270(84)90007-4]

Morris, R. G. (1981). Spatial localization does not require the presence of local cues. Learning and Motivation, 12(2), 239-60. [DOI:10.1016/0023-9690(81)90020-5] 
Neath, I., \& Surprenant, A. M. (2005). Mechanisms of memory. Handbook of Cognition. New York: SAGE Publications. [DOI:10.4135/9781848608177.n9]

Oliverio, A., \& Bovet, D. (1966). Effects of age on maze learning and avoidance conditioning of mice. Life Sciences, 5(14), 1317-24. [DOI:10.1016/0024-3205(66)90033-6]

Olton, D. S. (1979). Mazes, maps, and memory. American Psychologist, 34(7), 583-96. [DOI:10.1037/0003-066X.34.7.583] [PMID]

Olton, D. S., \& Samuelson, R. J. (1976). Remembrance of places passed: Spatial memory in rats. Journal of Experimental Psychology: Animal Behavior Processes, 2(2), 97-116. [DOI:10.1037/00977403.2.2.97]

Paul, C.M., Magda, G., \& Abel, S. J. B. B. R. (2009). Spatial memory: Theoretical basis and comparative review on experimental methods in rodents. Behavioural Brain Research, 203(2), 151-64. [DOI:10.1016/j.bbr.2009.05.022] [PMID]

Pellow, S., Chopin, P., File, S. E., \& Briley, M. (1985). Validation of open: closed arm entries in an elevated plus-maze as a measure of anxiety in the rat. Journal of Neuroscience Methods, 14(3), 149-67. [DOI:10.1016/0165-0270(85)90031-7]

Rosenfeld, C. S., \& Ferguson, S. A. J. J. O. V. E. J. (2014). Barnes maze testing strategies with small and large rodent models. Journal of Visualized Experiments, 101(1), 27-33. [DOI:10.3791/51194] [PMID] [PMCID]

Rolls, E. T. (2000). Memory systems in the brain. Annual Review of Psychology, 51, 599-30. [DOI:10.1146/annurev.psych.51.1.599] [PMID]

Scott, T. C. (1931). The Hampton Court maze. The Pedagogical Seminary and Journal of Genetic Psychology, 39(2), 287-9. [DOI: 10.1080/08856559.1931.10532311]

Shepard, J. F. (1920). Habit formation and higher mental capacities in animals. Psychological Bulletin, 17(6), 187-91. [DOI:10.1037/h0067986]

Simon, H. A. (1957). Amounts of fixation and discovery in maze learning behavior. Psychometrika, 22(3), 261-8. [DOI:10.1007/ BF02289126]

Small, W. S. (1901). Experimental study of the mental processes of the rat. II. The American Journal of Psychology, 12(2), 206-39. [DOI:10.2307/1412534]

Spangler, E. L., Chachich, M. E., Curtis, N. J., \& Ingram, D. K. (1989). Age-related impairment in complex maze learning in rats: Relationship to neophobia and cholinergic antagonism. Neurobiology of Aging, 10(2), 133-41. [DOI:10.1016/01974580(89)90022-5]

Stone, C. P. (1928). The reliability of rat learning scores obtained from a modified Carr maze. The Pedagogical Seminary and Journal of Genetic Psychology, 35(4), 507-21. [DOI:10.1080/0885655 9.1928.10532169]

Stone, C. P., \& Nyswander, D. B. (1927). The reliability of rat learning scores from the multiple-T maze as determined by four different methods. The Pedagogical Seminary and Journal of Genetic Psychology, 34(4), 497-524. [DOI:10.1080/08856559. 1927.10532397]

Stone, C. P., \& Sturman-Huble, M. (1927). Food vs. sex as incentives for male rats on the maze-learning problem. The American Journal of Psychology, 38(3), 403-8. [DOI:10.2307/1415008]
Tolman, E. C., Ritchie, B., \& Kalish, D. J. J. O. E. P. (1946). Studies in spatial learning. I. Orientation and the Short-Cut. 36(1), 13 [DOI:10.1037/h0053944] [PMID]

Trueblood, C. K. (1929). A tunnel maze. The Pedagogical Seminary and Journal of Genetic Psychology, 36(4), 581-3. [DOI:10.1080/08 856559.1929.10532215]

Tulving, E. (2002). Episodic memory: From mind to brain. Annual Review of Psychology, 53(1), 1-25. https://doi.org/10.1146/ annurev.psych.53.100901.135114

Vargas-López, V., Lamprea, M. R., \& Múnera, A. (2011). Characterizing spatial extinction in an abbreviated version of the Barnes maze. Behavioural Processes, 86(1), 30-8. [DOI:10.1016/j. beproc.2010.08.002] [PMID]

Vincent, S. B. (1915). The white rat and the maze problem: The introduction of a tactual control. Journal of Animal Behavior 5(3), 175-84. [DOI:10.1037/h0075779]

Vorhees, C. V. (1987). Maze learning in rats: A comparison of performance in two water mazes in progeny prenatally exposed to different doses of phenytoin. Neurotoxicology and Teratology, 9(3), 235-41. [DOI:10.1016/0892-0362(87)90008-0]

Vorhees, C. V., \& Williams, M. T. (2016). Cincinnati water maze: A review of the development, methods, and evidence as a test of egocentric learning and memory. Neurotoxicology and Teratology, 57, 1-19. [DOI:10.1016/j.ntt.2016.08.002] [PMID] [PMCID]

Walton, A. (1930). Demonstrational and experimental devices. The American Journal of Psychology, 42(1), 109-14. [DOI:10.2307/1414428]

Warden, C. (1929). A standard unit animal maze for general laboratory use. The Pedagogical Seminary and Journal of Genetic Psychology, 36(1), 174-6. [DOI:10.1080/08856559.1929.10533084]

Warden, C. J. (1923). Some factors determining the order of elimination of culs-de-sac in the maze. Journal of Experimental Psychology, 6(3), 192-210. [DOI:10.1037/h0072293]

Washburn, M. F. (1926). Hunger and speed of running as factors in maze learning in mice. Journal of Comparative Psychology 6(2), 181-7. [DOI:10.1037/h0075214]

Watson, J. B. (1914). A circular maze with camera lucida attachment. Journal of Animal Behavior, 4(1), 56-9. [DOI:10.1037/ h0072544]

Wenrick, J. E. (1935). Some effects of partial suprarenalectomy upon the learning of white rats in a water maze. Journal of Comparative Psychology, 20(2), 243-62. [DOI:10.1037/h0061439]

Xu, R. X., Grigoryev, N., Li, T.L., Bian, H.S., Zhang, R., \& Liu, X.Y. (2013). Development of hexagonal maze procedure for evaluating memory in rat. Biomedical Reports, 1(1), 134-8. [DOI:10.3892/br.2012.16] [PMID] [PMCID]

Yoshioka, J. G. (1929). What is maze learning for the rat? The Pedagogical Seminary and Journal of Genetic Psychology, 36(1) 51-8. [DOI:10.1080/08856559.1929.10533085] 
This Page Intentionally Left Blank 